\title{
Understanding the Prevalence of Prediabetes and Diabetes in Patients With Cancer in Clinical Practice: A Real-World Cohort Study
}

Dominik J. Ose, DrPH, MPH 1,2; Richard Viskochil, $\mathrm{PhD}^{2}$; Andreana N. Holowatyj, PhD2,3,4; Mikaela Larson, CCRP2,3; Dalton Wilson, BSc²; William A. Dunson Jr, MD²; Vikrant G. Deshmukh, PhD, JD, MS5; J. Ryan Butcher, MBA3; Belinda R. Taylor, CTR ${ }^{6}$; Kim Svoboda, CTR ; Jennifer Leiser, MD1; Benjamin Tingey, BSc ${ }^{1}$; Benjamin Haaland, PhD²,3; David W. Wetter, PhD2,3; Simon J. Fisher, MD, PhD7; Mia Hashibe, PhD'1,2; and Cornelia M. Ulrich, PhD2,3

\begin{abstract}
Background: This study aimed to understand the prevalence of prediabetes (preDM) and diabetes mellitus (DM) in patients with cancer overall and by tumor site, cancer treatment, and time point in the cancer continuum. Methods: This cohort study was conducted at Huntsman Cancer Institute at the University of Utah. Patients with a first primary invasive cancer enrolled in the Total Cancer Care protocol between July 2016 and July 2018 were eligible. Prevalence of preDM and DM was based on ICD code, laboratory tests for hemoglobin A1c, fasting plasma glucose, nonfasting blood glucose, or insulin prescription. Results: The final cohort comprised 3,512 patients with cancer, with a mean age of 57.8 years at cancer diagnosis. Of all patients, $49.1 \%(n=1,724)$ were female. At cancer diagnosis, the prevalence of preDM and DM was $6.0 \%(95 \% \mathrm{Cl}, 5.3 \%-6.8 \%)$ and $12.2 \%(95 \% \mathrm{Cl}, 11.2 \%-13.3 \%)$, respectively. One year after diagnosis the prevalence was $16.6 \%(95 \% \mathrm{Cl}, 15.4 \%-17.9 \%)$ and $25.0 \%$ (95\% Cl, 23.6\%-26.4\%), respectively. At the end of the observation period, the prevalence of preDM and DM was $21.2 \%(95 \% \mathrm{Cl}$, $19.9 \%-22.6 \%)$ and $32.6 \%(95 \% \mathrm{Cl}, 31.1 \%-34.2 \%)$, respectively. Patients with myeloma $(39.2 \% ; 95 \% \mathrm{Cl}, 32.6 \%-46.2 \%)$ had the highest prevalence of preDM, and those with pancreatic cancer had the highest prevalence of DM (65.1\%; $95 \% \mathrm{Cl}, 57.0 \%-72.3 \%)$. Patients who underwent chemotherapy, radiotherapy, or immunotherapy had a higher prevalence of preDM and DM compared with those who did not undergo these therapies. Conclusions: Every second patient with cancer experiences preDM or DM. It is essential to foster interprofessional collaboration and to develop evidence-based practice guidelines. A better understanding of the impact of cancer treatment on the development of preDM and DM remains critical.
\end{abstract}

J Natl Compr Canc Netw 2021;19(6):709-718 doi: $10.6004 /$ jnccn.2020.7653

${ }^{1}$ Department of Family and Preventive Medicine, School of Medicine,

${ }^{2}$ Huntsman Cancer Institute, and ${ }^{3}$ Department of Population Health Sciences, School of Medicine, Salt Lake City, Utah; ${ }^{4}$ Department of Medicine, Vanderbilt University Medical Center, Nashville, Tennessee; and ${ }^{5}$ Data Science Service, University of Utah, ${ }^{6}$ Huntsman Cancer Registry, University of Utah Hospital, and ${ }^{7}$ Department of Internal Medicine, School of Medicine, University of Utah, Salt Lake City, Utah.

\section{Background}

Cancer and diabetes are challenging health systems worldwide. In the United States, it is estimated that 5\% of the population (16.9 million people) has cancer. ${ }^{1}$ At the same time, $9.4 \%$ of the US population (30.3 million people) has diabetes mellitus (DM). In addition, 33.9\% of American adults (84.1 million people) are living with prediabetes (preDM), increasing their chances of developing $\mathrm{DM}^{2}$ Overall, cancer and DM are often diagnosed simultaneously, independent of age, suggesting a possible common underlying mechanism. ${ }^{3}$

The co-occurrence of cancer and DM raises significant health challenges. In patients with cancer, DM is associated with higher rates of complications, ${ }^{4,5}$ a higher risk for hospitalization, ${ }^{6,7}$ increased mortality, ${ }^{8-10}$ increased psychological distress, ${ }^{11}$ and decreased healthrelated quality of life. ${ }^{12-14}$ In fact, DM is a common cause of noncancer mortality in patients with cancer. ${ }^{15}$

Research on the relationship between cancer and DM has long been focused on DM and its role as a risk factor in the development of several different types of cancer. ${ }^{16-19}$ However, a recent study by Hwangbo et $\mathrm{al}^{20}$ suggested that this relationship may be bidirectional because the diagnosis of DM frequently occurs after cancer diagnoses. Still, the entire extent of the problem is not well understood. Only a few studies have addressed the prevalence of DM in patients with cancer worldwide ${ }^{21-23}$ and in the United States. ${ }^{24-27}$ Evidence for the prevalence of preDM in patients with cancer remains scarce. ${ }^{21}$

Moreover, most studies addressing the prevalence of preDM or DM in patients with cancer are either retrospective analyses that did not include clinical data (eg, hemoglobin Alc [HbAlc], glucose levels $)^{24,26}$ or clinical

See JNCCN.org for supplemental online content. 
studies with a small number of patients and a focus on one specific cancer. For both types of studies, the lack of cancer specifics (eg, first primary cancer), timing of DM onset, and/or treatment characteristics limit the comparability and clinical utility of their results.

This study examined the prevalence of preDM and DM in patients with cancer using a large, prospective cancer cohort. We incorporated clinical data to determine the prevalence of preDM and DM in patients with cancer overall and by tumor site, cancer treatment, and time point in the cancer continuum.

\section{Methods}

\section{Design and Population}

This cohort study used the Total Cancer Care (TCC) protocol at University of Utah (UofU) Huntsman Cancer Institute (HCI). TCC is the primary biobanking research protocol at HCI, which serves as a large centralized clinical data and tissue repository. Participants enrolled under this protocol agree to be observed throughout their lifetime and release medical data for research, including cancer occurring before enrollment. The study population at the time of this project included 5,865 men and women who enrolled in TCC between July 1, 2016, and July 31, 2018. Participants were recruited at local sites in Salt Lake City, Utah, including HCI and UofU hospitals and clinics. All patients provided informed consent. The HCI-TCC protocol was approved by the UofU Institutional Review Board (IRB \#89989).

\section{Cancer Characteristics}

All patients included in this analysis had an ICD-O diagnosis. ${ }^{28}$ Tumor behavior was determined based on ICD-O morphology codes. Only patients with a tumor behavior code of " 3 " (malignant, primary site) were included. Determination of whether a tumor was the first tumor was based on the sequence number, coded in the cancer registry. In this analysis, we included patients with the sequence number " 0 " (first and only tumor) and " 1 " (first of $>1$ tumor). Onset of cancer was defined as the date of the first ICD-O diagnosis documented in the Huntsman Cancer Registry (HCR), even if it was before enrollment in the TCC. The definition of tumor sites was based on the SEER Site Recode of ICD-O codes (ICD-O-3/WHO 2008 definition). ${ }^{7}$

\section{Determination of preDM and DM}

Criteria for determination of preDM and DM (Table 1) were based on American Diabetes Association (ADA) guidelines. ${ }^{29}$ Onset of preDM/DM was defined as the date of the first qualifying ICD code (preDM: ICD-9 790.xx and ICD-10 R73.xx; DM: ICD-9 250.xx and ICD-10 E8-E13) and/or by laboratory results (HbAlc: $5.7 \%-6.4 \%$

\begin{tabular}{|lcc|}
\hline \multicolumn{3}{|l}{ Table 1. Diagnostic Indicators for PreDM and DM } \\
\hline Indicator & PreDM & DM \\
\hline ICD-9 & $790 . x x$ & $250 . X X$ \\
\hline ICD-10 & R73.xx & E8-E13 \\
HbA1c (\%) & $5.7-6.4$ & $\geq 6.5$ \\
\hline Fasting plasma glucose (mg/dL) & $100-125$ & $\geq 126$ \\
\hline Blood glucose $(\mathrm{mg} / \mathrm{dL})^{\mathrm{a}}$ & $140-199$ & $\geq 200$ \\
\hline Prescriptions & - & Insulin \\
\hline
\end{tabular}

Abbreviations: DM, diabetes mellitus; HbA1c, hemoglobin A1c; preDM, prediabetes.

aTo confirm diagnosis, $\geq 2$ laboratory test results $>2$ days apart were required if no other indication of diagnosis was given (eg, ICD code, insulin prescription).

for preDM and $>6.4 \%$ for DM; fasting plasma glucose: $100-125 \mathrm{mg} / \mathrm{dL}$ for preDM and >125 mg/dL for DM; blood glucose: $140-199 \mathrm{mg} / \mathrm{dL}$ for preDM and >199 mg/ $\mathrm{dL}$ for $\mathrm{DM}$ ) and/or prescription for insulin. If the determination of DM or preDM was based solely on a laboratory result, then a second test in the respective range (preDM or DM), at least 3 days after the first test, was required to confirm the diagnosis.

\section{Data Collection}

Patients in this study were identified through the TCC Cancer Clinical Research database, which contains study information and is linked with related research and clinical information systems. For this analysis, we included data from the HCR and the UofU Health Enterprise Data Warehouse (EDW). Data from both sources (supplemental eTable 1, available with this article at JNCCN.org) were available for the period between January 2000 and July 2019 (for patients recruited between July 2016 and July 2018), allowing at least 1 year of followup for each patient.

\section{Exclusion Criteria}

Participants were excluded if they did not have a documented ICD-O diagnosis in the HCR, were not treated in the UofU health system (HCR "class of case" numbers $30,31,33,35,37,38,40-43,49$, and 99), were not diagnosed between January 2000 and December 2018, or were not between ages 18 and 90 years at cancer diagnosis, or if the cancer was not primary or not invasive (Figure 1).

\section{Statistical Analysis}

Clinicodemographic characteristics were examined by DM status (preDM, DM, or neither). Total counts and percentages among the DM status groups are displayed for categorical variables and means and standard deviations for quantitative variables (Table 1). Differences in clinicodemographics by DM status were examined using 


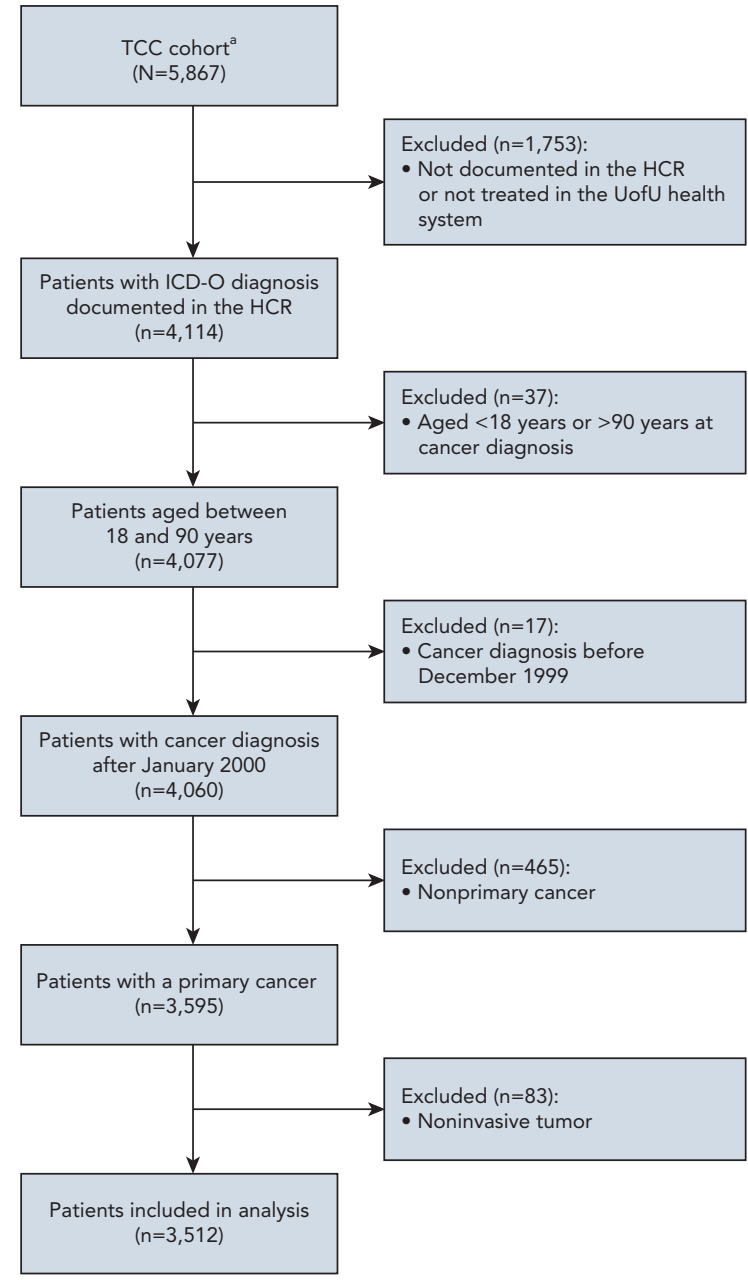

Figure 1. Flowchart with exclusion criteria.

Abbreviations: HCR, Huntsman Cancer Registry; TCC, total cancer care; UofU, University of Utah.

aParticipants in the TCC cohort as of July 31, 2018.

chi-square tests for categorical variables and one-way ANOVAs for continuous variables. Pairwise comparisons for characteristics by DM status (preDM vs neither, DM vs neither, DM vs preDM) were examined using chisquare tests for categorical variables and $t$ tests for continuous variables. The $P$ values from these pairwise comparisons were adjusted for multiplicity using the Hommel multiple comparison procedure. All statistical tests were 2 -sided, and $P$ values $<.05$ were considered statistically significant.

The prevalence of preDM and DM with 95\% confidence intervals was categorized by clinicodemographics, tumor site, therapy (surgery, chemotherapy, radiotherapy, hormone therapy, immunotherapy, glucocorticoids), diagnostic indicator (ICD code, laboratory test results, insulin prescription), and the time point within the cancer continuum (at or before cancer diagnosis, after cancer diagnosis). Patients were considered as having
$\mathrm{DM}$ or preDM at or before cancer diagnosis if they met DM or preDM criteria up to 2 weeks after cancer diagnosis. To complement these classifications, the time point "after cancer diagnosis" started 2 weeks after cancer diagnosis and was subdivided in "1 month after" (up to 30 days), "6 months after" (up to 182 days), "1 year after" (up to 365 days), and "end of the observation period" ( $>365$ days).

The length of follow-up was defined as the number of days between cancer diagnosis and the last documented date from data sources in the EDW (eg, encounter, ICD code, laboratory test). The median follow-up in months was provided. We constructed the $95 \%$ confidence intervals using the Wilson score confidence interval for binomial proportions. ${ }^{30}$ Analyses were conducted in $\mathrm{R}$ version 3.5.3 (R Foundation for Statistical Computing).

\section{Results}

\section{Description of the Cohort}

The HCI-TCC DM cohort (Figure 1) comprised 3,512 patients with cancer (median follow-up, 28 months), with a mean age of 57.8 years at cancer diagnosis. Of all patients, $49.1 \%(n=1,724)$ were female, $90.5 \%(n=3,177)$ were non-Hispanic White, $60.4 \%(n=2,122)$ were overweight or obese, and $70.2 \%(n=2,464)$ were Utah residents (Table 2).

Cancers of the breast $(13.8 \% ; n=484)$ and prostate $(14.1 \% ; n=494)$ were the most common (Table 3$)$. Regarding cancer severity and treatment, $31.2 \%(\mathrm{n}=1,096)$ of all patients had stage III/IV disease, $84.2 \%(\mathrm{n}=2,957)$ had undergone surgery, and $41.5 \%(n=1,458)$ had undergone chemotherapy (Table 4). In addition, $83.7 \%$ of patients $(\mathrm{n}=2,938)$ were treated with glucocorticoids (Table 2$)$.

Compared with patients with neither preDM nor $\mathrm{DM}$, those with preDM or DM were older $(P=.04$ and $P<.001$, respectively), more often underwent chemotherapy $(P<.001$, for both) or immunotherapy $(P<.001$ and $P=.005$, respectively), were more often treated with glucocorticoids $(P<.001$ and $P=.01$, respectively), and less frequently underwent surgery $(P<.001$, for both). Compared with patients with neither preDM nor DM, patients with DM were more often male $(P=.02)$ and more often Hispanic $(P=.001)$; patients with preDM more frequently received radiotherapy $(P=.002$; Table 2 , supplemental eTable 2).

\section{Prevalence by Clinicodemographics}

The prevalence of DM was higher in men (34.5\%; 95\% CI, $32.3 \%-36.7 \%)$ compared with women $(30.7 \%$; $95 \% \mathrm{CI}$, $28.6 \%-32.9 \%)$. Across body mass index categories, the prevalence of DM was $22.7 \%$ for patients with normal weight (95\% CI, 20.0\%-25.7\%), $28.0 \%$ for overweight (95\% CI, $25.3 \%-30.8 \%$ ), and $43.1 \%$ for obese patients (95\% CI, $40.2 \%-46.0 \%$ ) (supplemental eTable 3). 
Table 2. Clinicodemographic Characteristics Among Patients With Cancer

\begin{tabular}{|c|c|c|c|c|c|}
\hline & $\begin{array}{l}\text { Totala } \\
\text { n (\%) }\end{array}$ & $\begin{array}{l}\text { PreDM } \\
\text { n (\%) }\end{array}$ & $\begin{array}{l}\text { DM } \\
\text { n (\%) }\end{array}$ & $\begin{array}{l}\text { Neither PreDM } \\
\text { or DM } \mathbf{n}(\%)\end{array}$ & $P$ Value \\
\hline Study population & $3,512(100)$ & $745(21.2)$ & $1,145(32.6)$ & $1,622(46.2)$ & - \\
\hline Age-categorized ${ }^{b}$ & & & & & $<.001$ \\
\hline$<50 y$ & $854(24.3)$ & $194(26.0)$ & $206(18.0)$ & $454(28.0)$ & \\
\hline$\geq 80 \mathrm{y}$ & $127(3.6)$ & $20(2.7)$ & $53(4.6)$ & $54(3.3)$ & \\
\hline Sex & & & & & .02 \\
\hline Female & $1,724(49.1)$ & $360(48.3)$ & $529(46.2)$ & $835(51.5)$ & \\
\hline Male & $1,788(50.9)$ & $385(51.7)$ & $616(53.8)$ & 787 (48.5) & \\
\hline Non-Hispanic Asian & $38(1.1)$ & $7(0.9)$ & $15(1.3)$ & $16(1.0)$ & \\
\hline Non-Hispanic other ${ }^{c}$ & $142(4.0)$ & $26(3.5)$ & $54(4.7)$ & $62(3.8)$ & \\
\hline Hispanic & 137 (3.9) & $24(3.2)$ & $68(5.9)$ & $45(2.8)$ & \\
\hline $\mathrm{BMI}$, mean $(\mathrm{SD})^{\mathrm{d}}$ & $29.1(6.6)$ & $28.1(5.8)$ & $31.3(7.7)$ & $28.0(5.8)$ & $<.001$ \\
\hline$B M l^{d}$ & & & & & $<.001$ \\
\hline Underweight $\left(<18.5 \mathrm{~kg} / \mathrm{m}^{2}\right)$ & $33(0.9)$ & $7(0.9)$ & $9(0.8)$ & $17(1.0)$ & \\
\hline Normal $\left(18.5-24.9 \mathrm{~kg} / \mathrm{m}^{2}\right)$ & $814(23.2)$ & $198(26.6)$ & $185(16.2)$ & $431(26.6)$ & \\
\hline Overweight $(25.0-29.9$ kg/m²) & $1,004(28.6)$ & $222(29.8)$ & $281(24.5)$ & $501(30.9)$ & \\
\hline Obese $\left(\geq 30.0 \mathrm{~kg} / \mathrm{m}^{2}\right)$ & $1,118(31.8)$ & $210(28.2)$ & $482(42.1)$ & $426(26.3)$ & \\
\hline Utah & $2,464(70.2)$ & $557(74.8)$ & $818(71.4)$ & $1,089(67.1)$ & \\
\hline Idaho & $425(12.1)$ & $65(8.7)$ & $146(12.8)$ & $214(13.2)$ & \\
\hline Wyoming & $274(7.8)$ & $45(6.0)$ & $86(7.5)$ & $143(8.8)$ & \\
\hline Nevada & $168(4.8)$ & $39(5.2)$ & $54(4.7)$ & $75(4.6)$ & \\
\hline Other & $181(5.2)$ & $39(5.2)$ & $41(3.6)$ & $101(6.2)$ & \\
\hline Cancer stage & & & & & $<.001$ \\
\hline $0-1$ & $872(24.8)$ & $137(18.4)$ & $226(19.7)$ & $509(31.4)$ & \\
\hline II & $638(18.2)$ & $107(14.4)$ & $208(18.2)$ & 323 (19.9) & \\
\hline III & $553(15.7)$ & $94(12.6)$ & $167(14.6)$ & $292(18.0)$ & \\
\hline IV & $543(15.5)$ & $153(20.5)$ & $199(17.4)$ & $191(11.8)$ & \\
\hline Unknown/Not applicableg & $906(25.8)$ & $254(34.1)$ & $345(30.1)$ & 307 (18.9) & \\
\hline
\end{tabular}

(continued on next page)

Prevalence by Time Point

At cancer diagnosis, the prevalence of preDM was $6.0 \%(95 \%$ CI, $5.3 \%-6.8 \%)$ and $12.2 \%$ for DM (95\% CI, $11.2 \%-13.3 \%)$. One year after cancer diagnosis, the prevalence of preDM and DM was $16.6 \%$ (95\% CI, $15.4 \%-17.9 \%$ ) and $25.0 \%$ (95\% CI, $23.6 \%-26.4 \%)$, respectively. At the end of the observation period, it was $21.2 \%$ (95\% CI, $19.9 \%-22.6 \%$ ) and $32.6 \%$ (95\% CI, 31.1\%-34.2\%; Table 4), respectively. 


\begin{tabular}{|c|c|c|c|c|c|}
\hline & $\begin{array}{l}\text { Total }^{\mathrm{a}} \\
\text { n (\%) }\end{array}$ & $\begin{array}{c}\text { PreDM } \\
\text { n (\%) }\end{array}$ & $\begin{array}{l}\text { DM } \\
\text { n (\%) }\end{array}$ & $\begin{array}{c}\text { Neither PreDM } \\
\text { or DM } n(\%)\end{array}$ & $P$ Value \\
\hline \multicolumn{6}{|l|}{ Cancer treatment } \\
\hline Surgery & $2,957(84.2)$ & $594(79.7)$ & $945(82.5)$ & $1,418(87.4)$ & $<.001$ \\
\hline Chemotherapy & $1,458(41.5)$ & $425(57.0)$ & $548(48.0)$ & $485(29.9)$ & $<.001$ \\
\hline Radiotherapy & $1,035(29.5)$ & $251(33.7)$ & $349(30.5)$ & $435(26.8)$ & .002 \\
\hline Hormone therapy & $731(20.8)$ & $169(22.7)$ & $204(17.8)$ & $358(22.1)$ & .01 \\
\hline Immunotherapy & $339(9.7)$ & 99 (13.3) & $122(10.7)$ & $118(7.3)$ & $<.001$ \\
\hline Glucocorticoids & $2,938(83.7)$ & $656(88.1)$ & $972(84.9)$ & $1,310(80.8)$ & $<.001$ \\
\hline Cancer sequence number & & & & & $<.001$ \\
\hline 00 - only one primary cancer & $3,084(87.8)$ & $632(84.8)$ & $977(85.3)$ & $1,475(90.9)$ & \\
\hline 01 - multiple primary cancers & $428(12.2)$ & $113(15.2)$ & $168(14.7)$ & $147(9.1)$ & \\
\hline
\end{tabular}

Abbreviations: BMI, body mass index; DM, diabetes mellitus; preDM, prediabetes; RUCA, rural-urban commuting area.

aNot all percentages add up to 100 because of rounding decimal places.

${ }^{b}$ At cancer diagnosis.

'American Indian/Alaska Native, Hawaiian/other Pacific Islander, other, or unknown.

'BMI, at cancer diagnosis (90-day window before and after cancer diagnosis).

eDetermined from RUCA score on zip code.

fDetermined from last known residence.

gBrain and nervous system cancers are not routinely staged.

Prevalence by Diagnostic Indicator

Solely based on ICD codes, the prevalence of preDM and DM was $4.2 \%$ (95\% CI, 3.6\%-4.9\%) and $21.1 \%$ (95\% CI, $19.8 \%-22.5 \%)$, respectively, at the end of the observation period. Based on laboratory values, the prevalence was $20.6 \%$ (95\% CI, $19.3 \%-22.0 \%)$ and $22.2 \%$ (95\% CI, $20.9 \%-23.6 \%)$, respectively. With respect to insulin prescription, we observed a prevalence of $21.9 \%$ (95\% CI, $20.5 \%-23.3 \%$ ) for DM (Table 4 ).

\section{Prevalence by Cancer Treatment}

Compared with patients who did not undergo chemotherapy, those who did had a higher prevalence of preDM (29.1\% vs $15.6 \%)$ and DM (37.6\% vs $29.0 \%)$. The same was true in patients undergoing radiotherapy (preDM: $24.3 \%$ vs $19.9 \%$; DM: $33.7 \%$ vs $32.1 \%$ ), those undergoing immunotherapy (preDM: $29.2 \%$ vs $20.4 \%$; DM: $36.0 \%$ vs $32.2 \%$ ), and those receiving glucocorticoids (preDM: $22.3 \%$ vs $15.5 \%$; DM: $33.1 \%$ vs $30.1 \%$ ).

\section{Prevalence by Tumor Site}

There were vast differences in the prevalence of preDM and DM between tumor sites (Table 3), with the highest prevalence of preDM in patients with myeloma (39.2\%) and of DM in those with pancreatic cancer $(65.1 \%)$. The lowest prevalence of preDM and DM was observed among patients with cutaneous melanoma $(11.0 \%$ and $20.1 \%$, respectively) (Figure 2, Table 3).

\section{Discussion}

Our analysis had 3 major findings. First, in patients with cancer, preDM/DM are highly prevalent and every second patient is affected. Second, the prevalence of preDM/DM varies widely with respect to tumor site and cancer treatment. For example, in patients with pancreatic cancer and in those undergoing chemotherapy, the prevalence of DM is markedly higher than in the entire population of patients with cancer analyzed in this study. Third, the prevalence of preDM/DM increases substantially after cancer diagnosis. Consequently, there may be potential to prevent new onset of preDM/DM and the transition from preDM to DM.

At the time of cancer diagnosis, the prevalence of DM in our cohort (mean age, 58 years) was $12.2 \%$, a value very similar to the $12.7 \%$ DM prevalence reported by the CDC for adults aged 45 to 64 years. $^{2}$ However, the 2.5-fold increase in DM prevalence to $32.6 \%$ by the end of the observation period suggests very strongly that the cancer treatment markedly and pathologically increased the risk of the development of DM. This prevalence rate of $32.6 \%$ was much higher than a previously reported DM prevalence in patients with cancer. ${ }^{24}$

Interestingly, the prevalence of preDM in our cohort at the time of cancer diagnosis was $6.0 \%$, a value much lower than the expected preDM prevalence of $40.9 \%$ reported by the CDC for adults aged 45 to 64 years. ${ }^{2} \mathrm{We}$ list the reasons for this likely underestimation in the later discussion of the study's strengths and limitations. However, consistent with our finding that the prevalence of DM increased substantially after cancer diagnosis, the 2.6 -fold increase in preDM prevalence up to $21.2 \%$ by the end of the observation period is also consistent with the notion that cancer treatment markedly raises blood sugar levels and increases the risk for development of preDM.

Regarding specific cancer types, we observed a high prevalence of DM in patients with pancreatic cancer, 
Table 3. Prevalence of PreDM and DM at End of Observation Period

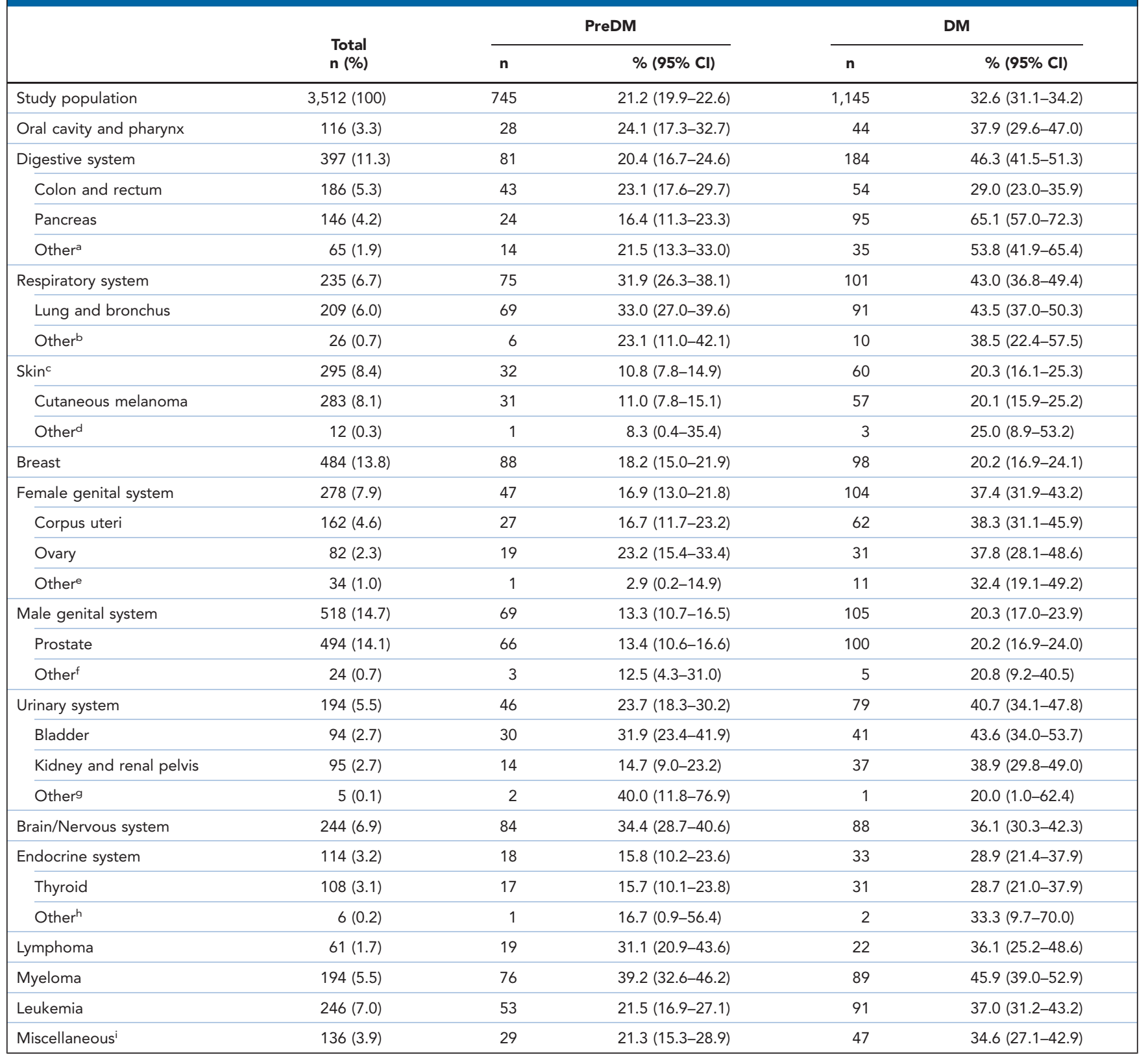

Abbreviations: DM, diabetes mellitus; preDM, prediabetes.

aEsophagus, stomach, small intestine, liver, intrahepatic bile duct, anus, gallbladder other biliary, retroperitoneum, peritoneum, other digestive organs.

${ }^{b}$ Nose, larynx, pleura, trachea, other.

'Excluding basal and squamous.

dOther nonepithelial, squamous cell carcinoma.

bladder cancer, and cancers of the respiratory system, whereas those with breast cancer, cutaneous melanoma, and prostate cancer had the lowest prevalence. Findings of previous studies have pointed in the same direction. . $1,27,31,32^{2}$ In contrast to earlier studies, our results provide a systematic overview of the prevalence of preDM and DM for various cancer types. ${ }^{33}$ For most of these cancer types, the prevalence of preDM has never been reported.
eCervix uteri, vagina, vulva, other genital organs.

fTestis, penis, other genital organs.

gUreter, other urinary organs.

hOther endocrine system organs.

'Kaposi sarcoma, mesothelioma, eye orbit, soft tissue, bone joints, other miscellaneous.

Cancer and DM are closely linked and share several risk factors, including male sex, older age, and obesity. ${ }^{34,35}$ In our study, patients with DM also tended to be older, more often male, and more often obese compared with those with cancer without DM. However, shared risk factors alone cannot explain the rapid increase of DM after cancer diagnosis. For example, in 2 earlier studies, the risk of developing DM after cancer 


\section{Table 4. Prevalence of PreDM and DM Among Patients With Cancer}

\begin{tabular}{|c|c|c|c|c|c|}
\hline & Total $\mathbf{n}$ & \multicolumn{2}{|c|}{ PreDM } & \multicolumn{2}{|c|}{ DM } \\
\hline Study population & 3,512 & 745 & $21.2(19.9-22.6)$ & 1,145 & $32.6(31.1-34.2)$ \\
\hline By time point ${ }^{\mathrm{a}}$ & 3,512 & & & & \\
\hline At cancer diagnosis ${ }^{b}$ & & 211 & $6.0(5.3-6.8)$ & 429 & $12.2(11.2-13.3)$ \\
\hline \multicolumn{6}{|l|}{ After cancer diagnosis } \\
\hline 6 mo after & & 536 & $15.3(14.1-16.5)$ & 824 & $23.5(22.1-24.9)$ \\
\hline $1 \mathrm{y}$ after & & 584 & $16.6(15.4-17.9)$ & 877 & $25.0(23.6-26.4)$ \\
\hline End of OP (>365 d) & & 745 & $21.2(19.9-22.6)$ & 1,145 & $32.6(31.1-34.2)$ \\
\hline By diagnostic indicator ${ }^{c, d}$ & 3,512 & & & & \\
\hline Based on ICD codes & & 147 & $4.2(3.6-4.9)$ & 742 & $21.1(19.8-22.5)$ \\
\hline DM other & & - & - & 232 & $6.6(5.8-7.5)$ \\
\hline Based on laboratory values ${ }^{e}$ & & 725 & $20.6(19.3-22.0)$ & 781 & $22.2(20.9-23.6)$ \\
\hline $\mathrm{HbA1c}$ & & 184 & $5.2(4.5-6.0)$ & 314 & $8.9(8.0-9.9)$ \\
\hline Fasting plasma glucose & & 25 & $0.7(0.5-0.1 .0)$ & 42 & $1.2(0.9-1.6)$ \\
\hline Blood glucose & & 745 & $21.2(19.9-22.6)$ & 732 & $20.8(19.5-22.2)$ \\
\hline Based on insulin prescription & & - & - & 768 & $21.9(20.5-23.3)$ \\
\hline \multicolumn{6}{|l|}{ By cancer treatment ${ }^{d}$} \\
\hline \multicolumn{6}{|l|}{ Chemotherapy } \\
\hline Yes & 1,458 & 425 & $29.1(26.9-31.5)$ & 548 & $37.6(35.1-40.1)$ \\
\hline No & 2,477 & 494 & $19.9(18.4-21.6)$ & 796 & $32.1(30.3-34.0)$ \\
\hline \multicolumn{6}{|l|}{ Hormone therapy } \\
\hline Yes & 731 & 169 & $23.1(20.2-26.3)$ & 204 & $27.9(24.8-31.3)$ \\
\hline No & 2,777 & 576 & $20.7(19.3-22.3)$ & 939 & $33.8(32.1-35.6)$ \\
\hline \multicolumn{6}{|l|}{ Immunotherapy } \\
\hline Yes & 339 & 99 & $29.2(24.6-34.3)$ & 122 & $36.0(31.1-41.2)$ \\
\hline No & 3,172 & 646 & $20.4(19.0-21.8)$ & 1,022 & $32.2(30.6-33.9)$ \\
\hline \multicolumn{6}{|l|}{ Glucocorticoids } \\
\hline Yes & 2,938 & 656 & $22.3(20.9-23.9)$ & 972 & 33.1 (31.4-34.8) \\
\hline No & 574 & 89 & $15.5(12.8-18.7)$ & 173 & $30.1(26.5-34.0)$ \\
\hline
\end{tabular}

Abbreviations: DM, diabetes mellitus; HbA1c, hemoglobin A1c; OP, observation period; preDM, prediabetes.

aSum of patients with preDM by time frame is 1,343. This number reflects the number of all patients who had preDM over time (lifetime prevalence). Because 598 patients transitioned to DM, at the end of the OP 745 patients had preDM.

bPrevalence as of 2 weeks after cancer diagnosis including all patients before cancer diagnosis.

cNumbers are not mutually exclusive. For example, a patient can have an ICD code for DM and an HbA1c in the DM range.

${ }^{d} A t$ the end of the OP.

ePreDM and DM diagnoses based solely on laboratory values were valid only if a patient had $\geq 2$ laboratory values $>2$ days apart to more fully establish robust diagnosis. 


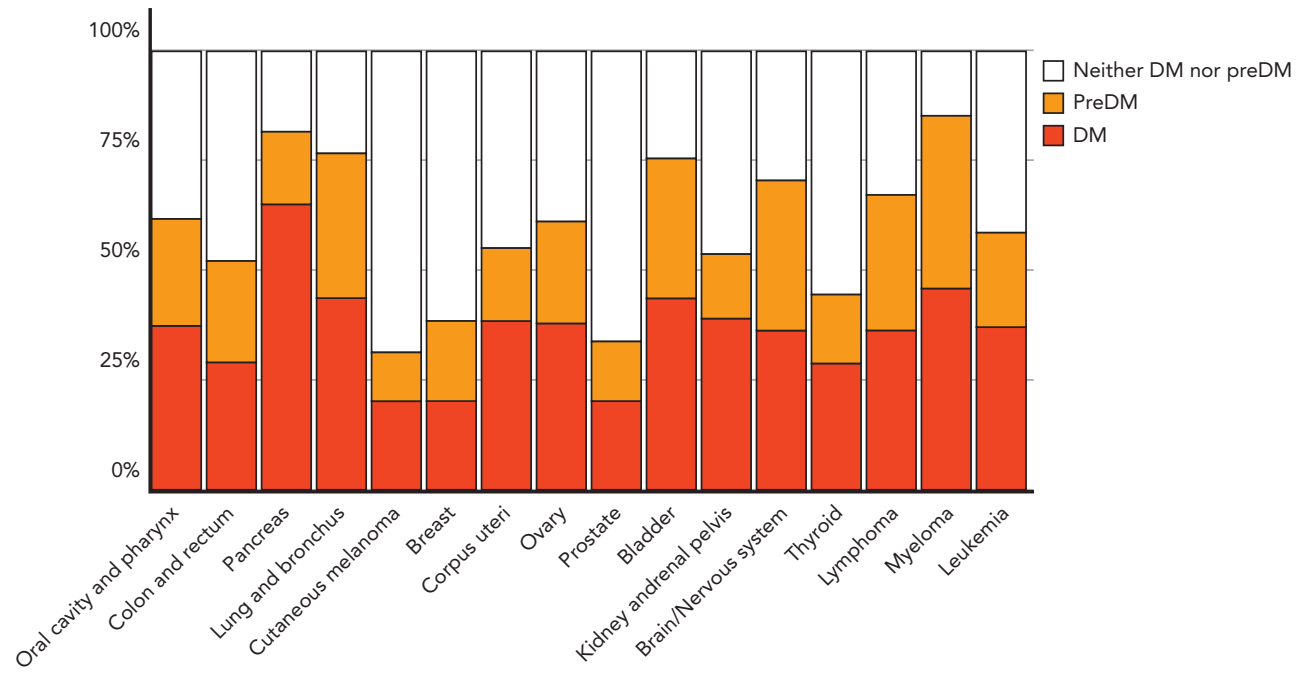

Figure 2. Patterns of preDM and DM among selected cancer types. Abbreviations: DM, diabetes mellitus; preDM, prediabetes.

diagnosis was similar in women and men, and younger patients were at higher risk compared with older patients. ${ }^{20,36}$

Instead, increasing evidence supports the impact of cancer treatment on the development of DM. In our study, patients undergoing chemotherapy, radiotherapy, immunotherapy, or treatment with glucocorticoids had a higher prevalence of DM compared with those not receiving those treatments. Previous research has indicated that treatment with antineoplastics is linked to hyperglycemia and DM by interfering with insulin production and secretion (eg, L-asparaginase, ${ }^{37,38}$ immune checkpoint inhibitors ${ }^{39-41}$ ), reducing insulin sensitivity (eg, nucleoside metabolic inhibitors, ${ }^{42,43}$ mTOR inhibitors ${ }^{44,45}$ ), or both (eg, selective estrogen receptor modulators ${ }^{46}$ ). Glucocorticoids, as part of cancer treatment or used to manage cancer treatment-related adverse effects, ${ }^{47,48}$ are also related to hyperglycemia in patients with cancer. ${ }^{49-51}$

Still, diagnosing and managing DM in patients with cancer remains a challenge. Glycemic control is frequently insufficient, ${ }^{26}$ and preDM and DM are frequently undiagnosed in patients with cancer. ${ }^{21}$ Often, providers and patients prioritize treatment of cancer over management of DM. ${ }^{52,53}$ In the absence of definitive guidelines, specific roles and responsibilities of managing DM and other chronic diseases remain unclear. ${ }^{54}$

This study has several strengths. The basis for this analysis is the HCI-TCC DM cohort, a large real-world population of patients with cancer from the western United States. Our population was restricted to patients diagnosed with a pathologically confirmed first primary invasive cancer. Through linkage of the HCI-TCC clinical data repository, the HCR, and the UofU Health EDW, a wealth of clinical and study-related data was available for this analysis. This is the first study to systematically analyze the prevalence of preDM and DM across cancer types.

This study also has several limitations. The prevalence of preDM and DM was based on clinical data and had been assessed based on ADA guidelines. ${ }^{29}$ However, even if glucose is measured several times during cancer treatment (eg, before surgery), systematic glucose monitoring is neither standard of care nor part of the HCI-TCC protocol. Therefore, the measurement of fasting or fed blood glucose levels alone may underestimate the true prevalence of preDM and DM.

In addition, because preDM as a formal diagnosis is often not coded (and is certainly underreported) and because glucose tolerance tests were not performed, the prevalence of preDM may have been underestimated. Because preDM and DM are often treated with various glucose-lowering drugs, restricting the diagnosis criteria to only one drug (insulin) may have also led to an underestimation of the prevalence of both preDM and DM. Finally, the ADA guidelines have not been specifically developed for patients with cancer. Whether elevated laboratory results, even if they are 2 days apart, are actually diagnostic for DM, perhaps only indicate stress-induced hyperglycemia, reflect the impact of cancer treatment (eg, glucocorticoid-induced preDM or DM), or are due to other factors, cannot be determined conclusively.

However, the diagnosis of DM was determined solely by laboratory values only in $8.9 \%(n=102)$ of all patients with $\mathrm{DM}(\mathrm{n}=1,145)$. In all other patients, there was 
at least an insulin prescription or a DM-related ICD code. Moreover, only 2.5\% $(n=29)$ of all patients with DM received glucocorticoids on the same days that their elevated glucose levels (in the DM range) were measured.

Because coding for preDM is often omitted, the portion of patients in whom preDM was determined based solely on laboratory values was higher. In $80.3 \%$ $(n=598)$ of all patients with preDM $(n=745)$, the definition of preDM was based solely on laboratory values. Among all patients with preDM, $27.2 \%(n=203)$ received glucocorticoids on the same days that their elevated glucose levels (in the preDM range) were measured. Overall, more detailed analyses are needed to examine the impact of glucocorticoids and chemotherapeutic agents on the development of new-onset DM and preDM after a cancer diagnosis.

\section{Conclusions}

PreDM and DM are highly prevalent in patients with cancer, and this prevalence increases markedly after diagnosis. To enable strategies for the prevention and management of DM during and after cancer treatment, it is essential to (1) recognize that patients with cancer are at high risk of developing DM and related complications (eg, hyperglycemia), (2) foster interprofessional collaboration between cancer treatment, endocrinology, and primary care, and (3) develop and implement evidence-based practice guidelines.

Submitted May 18, 2020; final revision received September 10, 2020; accepted for publication September 11, 2020.

Published online March 10, 2021.

Author contributions: Study concept and design: Ose, Viskochil, Holowatyj, Ulrich. Funding acquisition: Ose, Holowatyj, Haaland, Ulrich. Data acquisition: Larson, Wilson, Deshmukh, Butcher, Taylor, Svoboda. Statistical analysis: Tingey, Haaland. Data interpretation: Ose, Viskochil, Holowatyj, Dunson, Fisher. Manuscript preparation: Ose. Critical revision: Viskochil, Holowatyj, Larson, Wilson, Dunson, Deshmukh, Butcher, Taylor, Svoboda, Leiser, Tingey, Haaland, Wetter, Fisher, Hashibe, Ulrich. Administrative, technical, or material support: Larson. Supervision: Leiser, Wetter, Fisher, Hashibe, Ulrich.

Disclosures: Dr. Ulrich has disclosed that due to her position as Cancer Center Director, she oversees research funded by several pharmaceutical companies but has not received funding directly herself. The remaining authors have disclosed that they have not received any financial consideration from any person or organization to support the preparation, analysis, results, or discussion of this article.

Funding: This work was supported by a grant from Driving out Diabetes: A Larry H. Miller Family Wellness Initiative. Research reported in this work was also supported by the $\mathrm{NCl}$ of the NIH under award numbers U01 CA206110, R01 CA189184, and R01 CA207371 (C.M. Ulrich), and P30 CA042014.

Research reported in this work utilized the Cancer Biostatistics Shared Resource at the Huntsman Cancer Foundation at the University of Utah. Dr. Holowatyj was supported by the National Human Genome Research Institute of the NIH under Ruth L. Kirschstein National Research Service award number T32 HG008962.

Disclaimer: Driving out Diabetes: A Larry H. Miller Family Wellness Initiative had no role in the design and conduct of the study; the collection, management, analysis, and interpretation of the data; the preparation, review, or approval of the manuscript; and the decision to submit the manuscript for publication. The content is solely the responsibility of the authors and does not necessarily represent the official views of the NIH.

Correspondence: Dominik J. Ose, DrPH, MPH, Department of Family and Preventive Medicine, University of Utah, 375 Chipeta Way, Salt Lake City, UT 84108. Email: dominik.ose@hsc.utah.edu

\section{References}

1. National Cancer Institute, Division of Cancer Control \& Population Sciences. NCl Office of Cancer Survivorship. Accessed September 21, 2020. Available at: https://cancercontrol.cancer.gov/ocs

2. National Diabetes Statistics Report, 2017. Estimates of diabetes and its burden in the United States. Accessed September 21, 2020. Available at: https://dev.diabetes.org/sites/default/files/2019-06/cdc-statistics-report2017.pdf

3. Giovannucci E, Harlan DM, Archer MC, et al. Diabetes and cancer: a consensus report. Diabetes Care 2010;33:1674-1685.

4. Zaorsky NG, Shaikh T, Ruth K, et al. Prostate cancer patients with unmanaged diabetes or receiving insulin experience inferior outcomes and toxicities after treatment with radiation therapy. Clin Genitourin Cancer 2017;15:326-335.e3.

5. Raikundalia MD, Fang $\mathrm{CH}$, Spinazzi EF, et al. Impact of diabetes mellitus on head and neck cancer patients undergoing surgery. Otolaryngol Head Neck Surg 2016;154:294-299.

6. Karlin NJ, Kosiorek HE, Castro JC, et al. Risk of hospitalization in patients with diabetes mellitus who have solid-organ malignancy. Future Sci OA 2016;2:FSO129.

7. Dąbrowski M, Grondecka A. Diabetes as a risk factor of hospitalization in the surgical ward due to cancer in the elderly and middle-aged population. Arch Med Sci 2017;13:1025-1030.

8. Barone $\mathrm{BB}$, Yeh $\mathrm{HC}$, Snyder $\mathrm{CF}$, et al. Long-term all-cause mortality in cancer patients with preexisting diabetes mellitus: a systematic review and meta-analysis. JAMA 2008;300:2754-2764.

9. Barone BB, Yeh HC, Snyder CF, et al. Postoperative mortality in cancer patients with preexisting diabetes: systematic review and meta-analysis. Diabetes Care 2010;33:931-939.

10. Ranc K, Jørgensen ME, Friis $\mathrm{S}$, et al. Mortality after cancer among patients with diabetes mellitus: effect of diabetes duration and treatment. Diabetologia 2014:57:927-934.
11. Hoffman KE, McCarthy EP, Recklitis CJ, et al. Psychological distress in long-term survivors of adult-onset cancer: results from a national survey. Arch Intern Med 2009;169:1274-1281.

12. Thong MSY, van de Poll-Franse L, Hoffman RM, et al. Diabetes mellitus and health-related quality of life in prostate cancer: 5-year results from the Prostate Cancer Outcomes Study. BJU Int 2011;107:1223-1231.

13. Yabroff KR, Lawrence WF, Clauser $\mathrm{S}$, et al. Burden of illness in cancer survivors: findings from a population-based national sample. J Nat Cancer Inst 2004;96:1322-1330

14. Bowker SL, Pohar SL, Johnson JA. A cross-sectional study of healthrelated quality of life deficits in individuals with comorbid diabetes and cancer. Health Qual Life Outcomes 2006;4:17.

15. Shin DW, Ahn E, Kim H, et al. Non-cancer mortality among long-term survivors of adult cancer in Korea: national cancer registry study. Cancer Causes Control 2010;21:919-929.

16. Renehan A, Smith U, Kirkman MS. Linking diabetes and cancer: a consensus on complexity. Lancet 2010;375:2201-2202.

17. Vigneri P, Frasca F, Sciacca L, et al. Diabetes and cancer. Endocr Relat Cancer 2009:16:1103-1123.

18. Tsilidis KK, Kasimis JC, Lopez DS, et al. Type 2 diabetes and cancer: umbrella review of meta-analyses of observational studies. BMJ 2015;350: g7607.

19. Huang $Y$, Cai $X$, Qiu M, et al. Prediabetes and the risk of cancer: a metaanalysis. Diabetologia 2014;57:2261-2269.

20. Hwangbo $Y$, Kang $D$, Kang $M$, et al. Incidence of diabetes after cancer development: a Korean national cohort study. JAMA Oncol 2018;4 1099-1105.

21. Roeyen G, Jansen M, Chapelle $T$, et al. Diabetes mellitus and prediabetes are frequently undiagnosed and underreported in patients referred for pancreatic surgery. A prospective observational study. Pancreatology 2016;16:671-676. 
22. Lohmann AE, Ennis M, Taylor SK, et al. Metabolic factors, anthropometric measures, diet, and physical activity in long-term breast cancer survivors: change from diagnosis and comparison to non-breast cancer controls. Breast Cancer Res Treat 2017; 164:451-460.

23. Cetin M, Colak R, Bayram F, et al. High prevalence of diabetes in patients with pancreatic cancer in central Anatolia, Turkey. Diabetes Res Clin Pract 2002;58:97-100.

24. Edwards BK, Noone A-M, Mariotto AB, et al. Annual Report to the Nation on the status of cancer, 1975-2010, featuring prevalence of comorbidity and impact on survival among persons with lung, colorectal, breast, or prostate cancer. Cancer 2014;120:1290-1314.

25. Paulus YM, Riedel ER, Sabra MM, et al. Prevalence of diabetes mellitus in patients with newly evaluated papillary thyroid cancer. Thyroid Res 2014;7:7.

26. Karlin NJ, Dueck AC, Cook CB. Cancer with diabetes: prevalence, metabolic control, and survival in an academic oncology practice. Endocr Pract 2012;18:898-905.

27. Aggarwal G, Kamada P, Chari ST. Prevalence of diabetes mellitus in pancreatic cancer compared to common cancers. Pancreas 2013;42:198-201.

28. Fritz AG, Percy C, Jack A, et al, eds. International Classification of Diseases for Oncology: ICD-O, 3rd ed, 1st rev. Geneva, Switzerland: World Health Organization; 2013.

29. American Diabetes Association. 2. Classification and diagnosis of diabetes: standards of medical care in diabetes-2019. Diabetes Care 2019;42(Suppl 1):S13-28.

30. Agresti A, Coull BA. Approximate is better than "exact" for interval estimation of binomial proportions. The Amer Stat 1998;52:119-126.

31. Bluethmann SM, Mariotto AB, Rowland JH. Anticipating the "silver tsunami": prevalence trajectories and comorbidity burden among older cancer survivors in the United States. Cancer Epidemiol Biomarkers Prev 2016;25:1029-1036.

32. Roujun C, Yanhua Y, Bixun L. High prevalence of diabetes mellitus and impaired glucose tolerance in liver cancer patients: a hospital based study of 4610 patients with benign tumors or specific cancers. F1000 Res 2016; 5:1397.

33. National Cancer Institute, Surveillance, Epidemiology, and End Results Program. Site recode. Accessed September 21, 2020. Available at: https://seer.cancer.gov/siterecode/

34. Garg SK, Maurer H, Reed K, et al. Diabetes and cancer: two diseases with obesity as a common risk factor. Diabetes Obes Metab 2014;16:97-110.

35. Hawkins ML, Blackburn BE, Rowe K, et al. Endocrine and metabolic diseases among colorectal cancer survivors in a population-based cohort. J Natl Cancer Inst 2020;112:78-86.

36. Cho J, Kang D, Hwangbo Y, et al. Risk of diabetes associated with cancer development-in reply. JAMA Oncol 2019;5:429-430.

37. Flores-Calderón J, Exiga-Gonzaléz E, Morán-Villota S, et al. Acute pancreatitis in children with acute lymphoblastic leukemia treated with L-asparaginase. J Pediatr Hematol Oncol 2009;31:790-793.
38. Yoshida $\mathrm{H}$, Imamura $\mathrm{T}$, Saito $\mathrm{AM}$, et al. Protracted administration of $\mathrm{L}$-asparaginase in maintenance phase is the risk factor for hyperglycemia in older patients with pediatric acute lymphoblastic leukemia. PLoS One 2015;10:e0136428.

39. Stamatouli AM, Quandt Z, Perdigoto AL, et al. Collateral damage: insulindependent diabetes induced with checkpoint inhibitors. Diabetes 2018; 67:1471-1480

40. Tzoulis P, Corbett RW, Ponnampalam S, et al. Nivolumab-induced fulminant diabetic ketoacidosis followed by thyroiditis. Endocrinol Diabetes Metab Case Rep 2018;2018:18-0111.

41. Godwin JL, Jaggi S, Sirisena I, et al. Nivolumab-induced autoimmune diabetes mellitus presenting as diabetic ketoacidosis in a patient with metastatic lung cancer. J Immunother Cancer 2017;5:40.

42. Michie CO, Sakala M, Rivans I, et al. The frequency and severity of capecitabine-induced hypertriglyceridaemia in routine clinical practice: a prospective study. Br J Cancer 2010;103:617-621.

43. Feng JP, Yuan XL, Li M, et al. Secondary diabetes associated with 5-fluorouracil-based chemotherapy regimens in non-diabetic patients with colorectal cancer: results from a single-centre cohort study. Colorectal Dis 2013;15:27-33.

44. Milluzzo A, Tumminia A, Vella $V$, et al. Short-term adverse effects of anticancer drugs in patients with type 2 diabetes. J Chemother 2019;31:150-159.

45. Gallo M, Muscogiuri G, Felicetti F, et al. Adverse glycaemic effects of cancer therapy: indications for a rational approach to cancer patients with diabetes. Metabolism 2018;78:141-154.

46. Lipscombe LL, Fischer HD, Yun L, et al. Association between tamoxifen treatment and diabetes: a population-based study. Cancer 2012;118: 2615-2622.

47. Yennurajalingam S, Frisbee-Hume S, Palmer JL, et al. Reduction of cancerrelated fatigue with dexamethasone: a double-blind, randomized, placebo-controlled trial in patients with advanced cancer. J Clin Oncol 2013;31:3076-3082

48. Wooldridge JE, Anderson CM, Perry MC. Corticosteroids in advanced cancer. Oncology (Williston Park) 2001;15:225-234.

49. Hwangbo $Y$, Lee EK. Acute hyperglycemia associated with anti-cancer medication. Endocrinol Metab (Seoul) 2017;32:23-29.

50. Kwon S, Hermayer KL, Hermayer K. Glucocorticoid-induced hyperglycemia. Am J Med Sci 2013;345:274-277.

51. Clore JN, Thurby-Hay L. Glucocorticoid-induced hyperglycemia. Endocr Pract 2009;15:469-474.

52. Goebel J, Valinski S, Hershey DS. Improving coordination of care among healthcare professionals and patients with diabetes and cancer. Clin J Oncol Nurs 2016;20:645-651.

53. Piette JD, Kerr EA. The impact of comorbid chronic conditions on diabetes care. Diabetes Care 2006;29:725-731.

54. Walsh J, Young JM, Harrison JD, et al. What is important in cancer care coordination? A qualitative investigation. Eur J Cancer Care (Engl) 2011; 20:220-227.

\section{See JNCCN.org for supplemental online content.}


Supplemental online content for:

\section{Understanding the Prevalence of Prediabetes and Diabetes in Patients With Cancer in Clinical Practice: A Real-World Cohort Study}

Dominik J. Ose, DrPH, MPH; Richard Viskochil, PhD; Andreana N. Holowatyj, PhD; Mikaela Larson, CCRP; Dalton Wilson, BSc; William A. Dunson Jr, MD; Vikrant G. Deshmukh, PhD, JD, MS; J. Ryan Butcher, MBA; Belinda R. Taylor, CTR; Kim Svoboda, CTR; Jennifer Leiser, MD; Benjamin Tingey, BSc; Benjamin Haaland, PhD; David W. Wetter, PhD; Simon J. Fisher, MD, PhD; Mia Hashibe, PhD; and Cornelia M. Ulrich, PhD

J Natl Compr Canc Netw 2021;19(6):709-718

eTable 1: Data Description

eTable 2: Pairwise Comparisons of Clinicodemographic Characteristics Among Patients With Cancer eTable 3: Prevalence of Prediabetes and Diabetes Mellitus Among Patients With Cancer 


\section{eTable 1. Data Description}

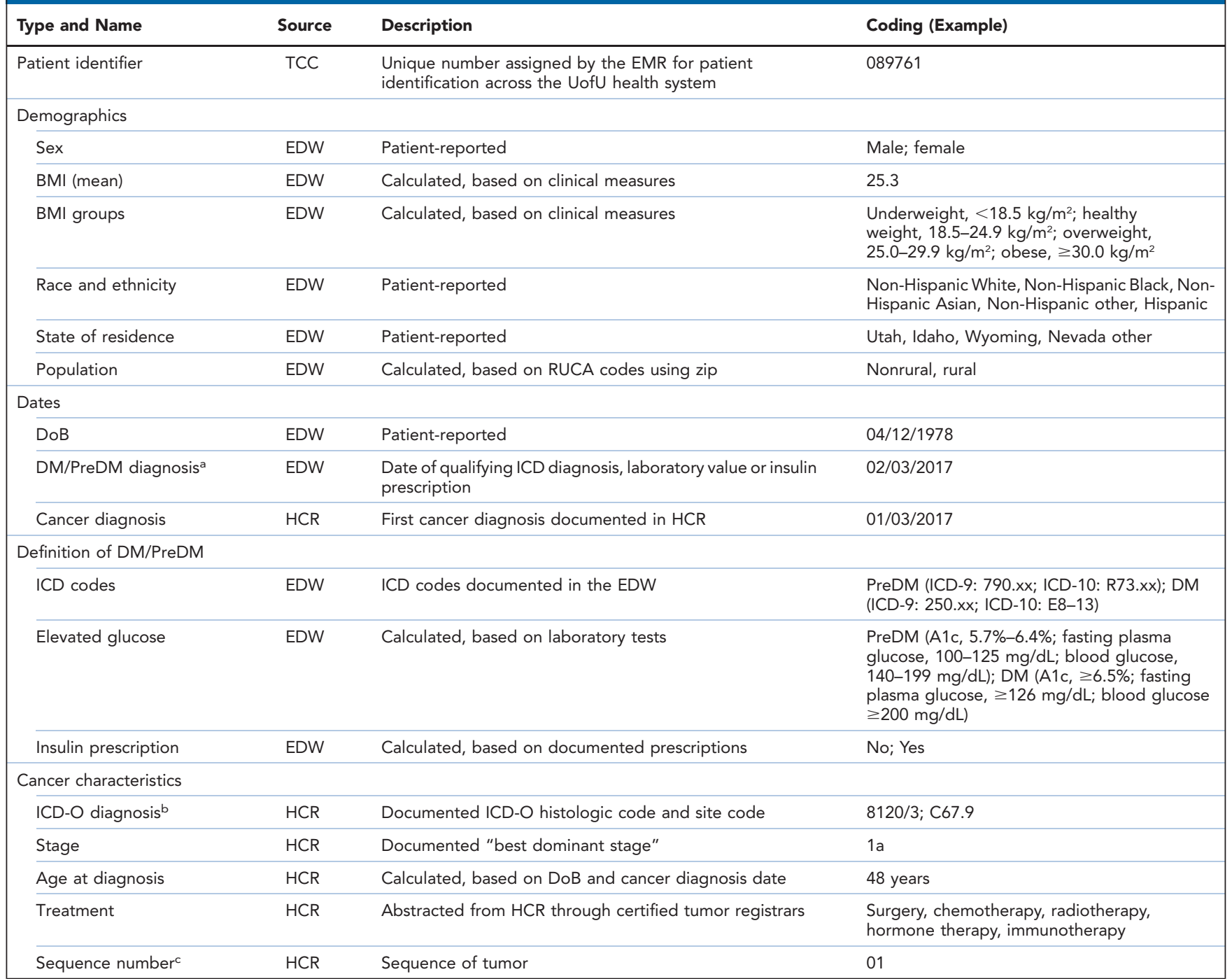

Abbreviations: BMI, body mass index; DM, diabetes mellitus; DoB, date of birth; EDW, Enterprise Data Warehouse; EMR, electronic medical record; HCR, Huntsman Cancer Registry; preDM, prediabetes; RUCA, rural-urban commuting area; TCC, Total Cancer Care; UofU, University of Utah.

aTo confirm diagnosis, $\geq 2$ laboratory test results $>2$ days apart were required if no other indication of diagnosis was given (ICD code, insulin prescription).

bRestricted /3 (invasive cancer, primary site).

cRestricted to 0 or 1 (primary cancers). 
eTable 2. Pairwise Comparisons of Clinicodemographic Characteristics Among Patients With Cancer: HCl-TCC DM, 2000-2019

\begin{tabular}{|c|c|c|c|}
\hline & \multicolumn{3}{|c|}{$P$ Values ${ }^{a}$} \\
\hline Age, mean & .04 & $<.001$ & $<.001$ \\
\hline Age, categorized & .052 & $<.001$ & $<.001$ \\
\hline Sex & .33 & .02 & .39 \\
\hline Race/Ethnicity & .10 & .001 & .001 \\
\hline BMI, categorized & .89 & $<.001$ & $<.001$ \\
\hline Population & .40 & .47 & .67 \\
\hline State of residence & .002 & .02 & .02 \\
\hline Cancer stage & $<.001$ & $<.001$ & .04 \\
\hline \multicolumn{4}{|l|}{ Cancer treatment } \\
\hline Hormone therapy & .79 & .02 & .02 \\
\hline Immunotherapy & $<.001$ & .005 & .10 \\
\hline Glucocorticoids & $<.001$ & .01 & .06 \\
\hline Cancer sequence & $<.001$ & $<.001$ & .82 \\
\hline
\end{tabular}

Abbreviations: BMI, body mass index; DM, diabetes mellitus; HCI-TCC, Huntsman Cancer Institute Total Cancer Care; preDM, prediabetes. ${ }^{a} P$ values adjusted by Hommel's multiple comparison procedure. 


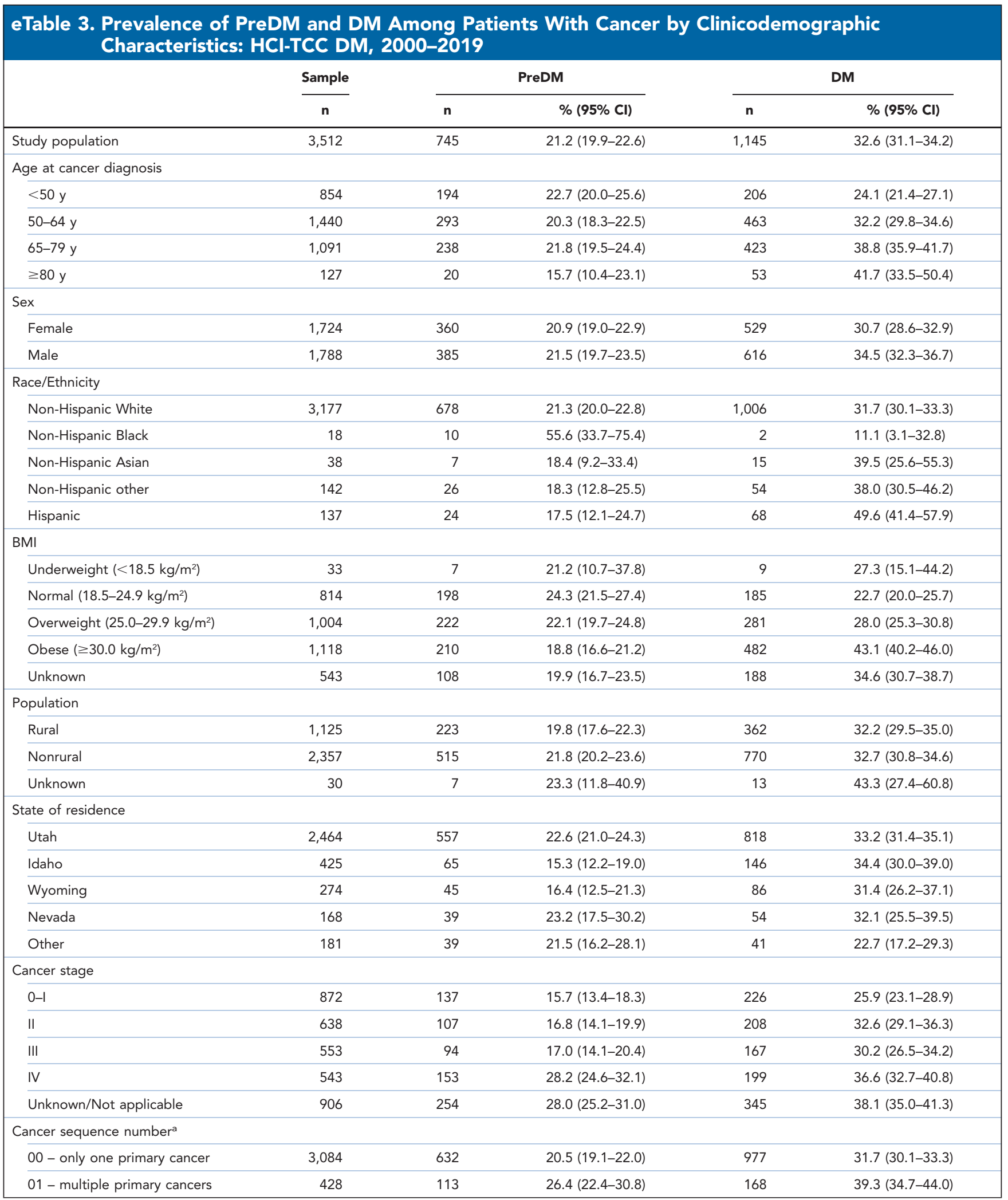

Abbreviations: $\mathrm{BMI}$, body mass index; DM, diabetes mellitus; preDM, prediabetes. aUsing row percents. 